\title{
IMPACT OF COVID-19 ON THE DIAGNOSIS AND MANAGEMENT OF MULTIPLE MYELOMA: EXPERIENCE FROM A CANADIAN CENTER
}

\author{
Victor H. Jimenez-Zepeda ${ }^{1,2, *}$, Patrick Yau ${ }^{1}$, Douglas Stewart ${ }^{1}$, Jowher Berhan ${ }^{1}$, Carole $_{\text {Chambers }}{ }^{1}$, \\ Holly Lee ${ }^{1}$, Jason Tay ${ }^{1,2}$, Peter Duggan ${ }^{1,2}$, Sylvia McCulloch ${ }^{1}$, Paola Neri ${ }^{1,2}$, and Nizar Bahlis ${ }^{1,2}$ \\ ${ }^{1}$ Department of Medical Oncology and Hematology, Tom Baker Cancer Center, Calgary, Alberta; \\ ${ }^{2}$ Charbonneau Cancer Research Institute, Calgary, Alberta, Canada
}

\begin{abstract}
Background: The impact of coronavirus disease-19 on the management of multiple myeloma (MM) has been recognized. However, the real effect on clinical outcomes remains poorly understood. Objective: We describe a local experience of the management of MM patients and report their outcomes during the current pandemic. Methods: All consecutive symptomatic MM patients seen at our center since 03/20 were evaluated. Results: A cohort of 156 patients diagnosed from 01/19 to 12/20 was analyzed to interrogate differences in presentation patterns. A total of $553 \mathrm{MM}$ patients were seen and/or treated at Tom Baker Cancer Center in the year of 2020. From those, $47.1 \%(n=261)$ were tested for severe acute respiratory syndrome coronavirus-2 (SARS-CoV-2). Sixteen patients tested positive and data are presented. In addition, a decrease of $21.7 \%$ in the rate of new smoldering MM/MM diagnosis was observed in 2020 as compared to 2019. Further, an increase in deaths was also observed in 2020. Conclusions: Our study confirms an increase lethality for MM patients infected with SARS-CoV-2. A balance between safety and need for cancer control should be emphasized. (REV INVEST CLIN. 2022;74(1):16-22)
\end{abstract}

Keywords: Myeloma. SARS-CoV-2. COVID-19.

\section{INTRODUCTION}

Severe acute respiratory syndrome coronavirus (SARS-CoV-2) is the novel human coronavirus responsible for the coronavirus disease 2019 (COVID-19) pandemic. On March 11, 2020, the World Health Organization declared COVID-19 as a pandemic. Globally, there have been 184,324,026 confirmed cases of COVID-19, including 3,992,680 deaths, reported to the World Health Organization (https://covid19. who.int) in 223 countries around the world. COVID-19 is a serious health threat, and risk varies between the different regions of the world. In Alberta, 232,269 patients have been confirmed with COVID-19 and 94,604 of them reside in Calgary.

Since December 2019, when COVID-19 emerged in Wuhan City and rapidly spread throughout China and around the world, data have been needed to understand better its impact on patients with cancer and
*Corresponding author:

Victor $\mathrm{H}$. Jimenez-Zepeda

E-mail: victor.zepeda@albertahealthservices.ca
Received for publication: 07-07-2021

Approved for publication: 28-07-2021

DOI: $10.24875 / R I C .21000347$

0034-8376 / (c) 2021 Revista de Investigación Clínica. Published by Permanyer. This is an open access article under the CC BY-NC-ND license (http://creativecommons.org/licenses/by-nc-nd/4.0/). 
in particular, to those with plasma cell disorders ${ }^{1}$. Patients with cancer are generally more susceptible to infections as compared to healthy people because they exhibit a systemic state of immunosuppression that is exacerbated during chemotherapy or radiotherapy ${ }^{2}$. When comparing non-cancer patients with those suffering from malignant tumors, the latter group is at a higher risk of developing a more severe infection and health deterioration is accelerated even after adjusting for age ${ }^{2}$. Multiple myeloma (MM) is a plasma cell neoplasm associated to an important degree of immunosuppression ${ }^{3}$. In addition, the use of chemotherapy, immunotherapy, and steroids plays a key role in increasing the morbidity and mortality associated to infections for MM cases, and thus, there is a concern among the myeloma community on the potential lethality risk for patients with MM infected with the novel SARS-CoV-2. A recent international effort reported data on 650 patients with plasma cell disorders from 10 different countries that were infected by the novel SARS-CoV-2 virus ${ }^{4}$. This study identified $33 \%$ of MM patients who died and a multivariate analysis demonstrated that age, high-risk MM, renal disease, and suboptimal $\mathrm{MM}$ control remained independent predictors of adverse outcome for MM patients suffering from COVID-19 infection. A recent study from Spain also found a higher mortality rate in MM patients with COVID-19 (34\%) compared with age- and sex-matched non-MM patients with COVID-19 $(23 \%)^{5}$. Based on the above mentioned, we aimed to assess the impact of COVID-19 in the management of $\mathrm{MM}$ in a comprehensive treating center for plasma cell disorders in Canada.

\section{METHODS}

MM patients included in the Clinical Data Integration Project and treated at the Tom Baker Cancer Center (TBCC) were evaluated. Briefly, this project included a cohort of patients over the age of 18 with a reportable hematological cancer from January 2004 to present day. Data from all patients diagnosed with $\mathrm{MM}$ in recent years were used to assess diagnosis patterns and mortality rates.

A cohort of consecutive symptomatic newly diagnosed MM patients from January 2019 to December 2020 was evaluated aiming to assess presentation patterns at the time of diagnosis. In addition, a retrospective chart review of the Alberta provincial Pharmacy Database was performed to analyze dispensing patterns in 2019 and 2020. Approval for the review of these records was obtained from the TBCC Institutional Review Board and informed consent was also obtained.

\section{Statistics}

Patient demographic variables were summarized by appropriate central measures of tendency and dispersion. Two-sided Fisher's exact test was used to test for differences between categorical variables. $p<$ 0.05 was considered statistically significant. Survival curves were constructed according to the KaplanMeier method and compared using the log rank test. All statistical analyses were performed using the SPSS 24.0 software.

\section{RESULTS}

A total of 356 patients with smoldering MM/MM (SMM/MM) were diagnosed from January 2017 to December 2020. Symptomatic MM was diagnosed in 324 patients in the same time period. We used a cohort of 156 patients diagnosed from January 2019 to December 2020 to evaluate the presentation patterns. Patients diagnosed in 2020 exhibited a trend toward higher number of bone marrow monoclonal plasma cells ( $50 \%$ vs. $30 \%, p=0.01$ ) and an increase of extramedullary disease ( $10.2 \%$ vs. $2.2 \%, p=0.02$ ) as compared to those diagnosed in 2019 (Table 1). The rest of clinical characteristics appeared to be similar regardless of the year of MM diagnosis. A decrease of $21.7 \%$ in the rate of new SMM/MM diagnosis and $22.8 \%$ for symptomatic MM were observed in 2020 as compared to 2019.

\section{Telemedicine and diagnostic investigations}

After March 11, 2020, when the WHO declared the pandemic, the cancer center initiated a telemedicine program. All MM patients seen at the cancer center from January to February of 2020 were assessed as in-person clinic visit. In March 2020, 24\% of MM patients were assessed virtually (92 out of 382 visits), increasing to $70 \%$ in April of 2020 (377 out of 536 visits). All virtually assessed patients were contacted 
Table 1. Clinical characteristics of MM patients seen at TBCC from January 2019 to December 2020

\begin{tabular}{|c|c|c|c|}
\hline Characteristic & $\begin{array}{l}\text { MM patients diagnosed } \\
\text { in 2020, } \\
(n=68)\end{array}$ & $\begin{array}{c}\text { MM patients diagnosed } \\
\text { in } 2019 \\
(n=88)\end{array}$ & $\mathrm{p}$-value \\
\hline $\begin{array}{l}\text { Median age, years } \\
\text { (range) }\end{array}$ & $\begin{array}{c}67.7 \\
(30-88)\end{array}$ & $\begin{array}{c}67.2 \\
(35-88)\end{array}$ & 0.4 \\
\hline \multicolumn{4}{|l|}{ Gender, \% } \\
\hline Female & 44 & 31 & 0.6 \\
\hline Male & 66 & 69 & \\
\hline Median Hb, g/L & 106 & 109 & 0.2 \\
\hline Median calcium, mmol/L & 2.4 & 2.39 & 0.5 \\
\hline Median creatinine, $\mu \mathrm{mol} / \mathrm{L}$ & 103.5 & 98.5 & 0.4 \\
\hline Median B2M, mg/L & 3.81 & 3.88 & 0.4 \\
\hline Median albumin, $\mathrm{g} / \mathrm{L}$ & 32 & 32.5 & 0.8 \\
\hline \multicolumn{4}{|l|}{ ISS stage, n (\%) } \\
\hline I & $\begin{array}{c}14 \\
(20.5)\end{array}$ & $\begin{array}{c}19 \\
(21.5)\end{array}$ & 0.4 \\
\hline II & $\begin{array}{c}27 \\
(39.7)\end{array}$ & $\begin{array}{c}40 \\
(45.4)\end{array}$ & \\
\hline III & $\begin{array}{l}26 \\
(38.2)\end{array}$ & $\begin{array}{c}24 \\
(27.2)\end{array}$ & \\
\hline Unknown & $\begin{array}{l}1 \\
(1.4)\end{array}$ & $\begin{array}{l}5 \\
(5.6)\end{array}$ & \\
\hline Median LDH, U/L & 182 & 180.5 & 0.2 \\
\hline Median BMPC, \% & 50 & 31 & 0.01 \\
\hline \multicolumn{4}{|l|}{ FISH, n (\%) } \\
\hline High risk* & $\begin{array}{l}11 \\
(16.1)\end{array}$ & $\begin{array}{c}17 \\
(19.3)\end{array}$ & 0.6 \\
\hline Standard risk & $\begin{array}{l}55 \\
(80)\end{array}$ & $\begin{array}{c}57 \\
(64.7)\end{array}$ & \\
\hline Unknown & $\begin{array}{l}2 \\
(2.9)\end{array}$ & $\begin{array}{l}14 \\
(15)\end{array}$ & \\
\hline \multicolumn{4}{|l|}{ Clinical presentation, \% } \\
\hline Extramedullary disease & 10.2 & 2.2 & 0.021 \\
\hline $\begin{array}{l}\text { Hypercalcemia } \\
(>2.75 \mathrm{mmol} / \mathrm{L})\end{array}$ & 4.7 & 12.5 & 0.6 \\
\hline $\begin{array}{l}\text { Creatinine } \\
\qquad(>177 \mu \mathrm{mol} / \mathrm{L})\end{array}$ & 22 & 22 & 0.9 \\
\hline Plasma cell leukemia & 1.4 & 1.1 & 0.6 \\
\hline Early death & 8.8 & 11.3 & 0.6 \\
\hline
\end{tabular}

*High risk was defined as the presence of $\mathrm{t}(4 ; 14)$, $\mathrm{t}(14 ; 16)$, or $17 \mathrm{p}$ deletion; Hb: hemoglobin; B2M: beta-2 microglobulin; BMPC: bone marrow plasma cell; FISH: fluorescence in situ hybridization; TBCC: Tom Baker Cancer Center.

by phone, and laboratory testing was restricted to local laboratory facilities where the immunocompromised patients were offered reserved spots by the
Alberta Health Services. As the first pandemic wave improved, in-person appointments increased. However, with the advent of the second COVID-19 wave, 
up to $60 \%$ of cases were assessed virtually (380 out of 626 visits in December of 2020). All patients not on active treatment were evaluated with phone consults, and laboratory testing was delayed at the peak of the outbreak.

\section{COVID-19 testing and COVID-19 infections among patients with MM seen and treated at TBCC}

The province of Alberta has one of the highest testing rates in the world. There have been $4,719,800$ tests completed for 2,195,190 people of a total population of approximately 4.37 million. COVID-19 testing was performed according to the Alberta Health Services guidelines. Further, a retrospective chart review of $597 \mathrm{MM}$ patients was conducted. A total of $553 \mathrm{MM}$ patients were seen and/or treated at TBCC in the year of 2020. From those, $47.1 \%(n=261)$ of cases were tested for SARS-CoV2 with $>30 \%$ of cases being tested multiple times. Sixteen patients tested positive for SARS-CoV2 (6.1\% of all tested patients $-16 / 261$ and $2.8 \%$ of the entire cohort of MM cases seen at TBCC in 2020). Clinical characteristics and outcomes are presented in Table 2. Briefly, 2 SMM and 14 symptomatic MM were positive for SARS-CoV-2. Eleven patients required admission to a hospital while three were admitted to intensive care units. Nine patients were receiving treatment with immunomodulatory drugs (ImIDs), three with proteasome inhibitors (PI), and six with daratumumab-based regimens at the time of COVID-19 infection. Patients with SMM were on observation only. Ten patients suffered from COVID-19 pneumonia. As a result of COVID-19, four patients died (25\%), three with symptomatic MM, and one with SMM.

\section{COVID-19 AND STEM CELL TRANSPLANTATION}

A total of 39 patients with MM received an autologous stem cell transplant in 2020. This was similar to that reported from 2017 to 2019. All patients undergoing autologous stem cell transplant in 2020 were screened for SARS-CoV-2 (laboratory testing) on admission to the hospital and before stem cell transplantation. No patients had autologous stem cell transplant delayed or denied due to a positive test, and no increase in transplant-related mortality was observed.

\section{COVID-19 and clinical trials}

TBCC is one of many Canadian Centers with an extensive experience in treating MM on clinical trials. In 2020, 11 clinical trials were open for recruitment; this was similar to those of 2019. In total, 35 MM patients were included in any of these clinical trials in 2020, in contrast to those seen in 2019 where $42 \mathrm{MM}$ patients were enrolled in clinical trials ( $16.6 \%$ fewer recruitments of MM patients for clinical trials).

\section{Treatment dispensation patterns}

Standard MM treatment data at the TBCC were collected using BDM Pharmacy v10.29.4 and Aria MO v13.7.2 computer systems. Patients who underwent standard myeloma treatments during the COVID-19 first wave (March 1-July 31, 2020) and second wave (August 1-December 31, 2020) were compared to the same time frame from the previous year in 2019. There were more myeloma patients who required standard therapy in the COVID-19 first wave, March 1-July 31, 2020, compared to the same time in 2019 (385 vs. 330). A similar increase was seen in the COVID-19 second wave, August 1-December 31, 2020, compared to the same time frame in 2019 (412 vs. 374). Corticosteroid use was similar between each of the first and second waves with their 2019 comparator time frame. No differences in corticosteroid dose changes were noticed. Treatment regimen changes were similar in the COVID-19 first wave and its 2019 comparator ( $21.6 \%$ vs. $21.5 \%$ ) ( $p$ $=0.6)$. Similar changes were seen with the second wave and its 2019 comparator ( $14.6 \%$ vs. $12.6 \%$ ) ( $p$ $=0.5$ ). There were no changes in medication route administration in 2020 compared to 2019 .

Based on this data, no changes in patterns of treatment or increase in relapsed MM cases were noted (ARIA-MO database data for progression) in 2020 as compared to 2019.

\section{Survival outcomes}

To assess the mortality rate since the pandemic initiation, we evaluated $886 \mathrm{MM}$ patients diagnosed from January 2011 to December 2020. From March 2020 to January 2021, 57 MM patients have died. Twenty-three patients died of myeloma-related complications, 10 died of non-myeloma-related causes, 


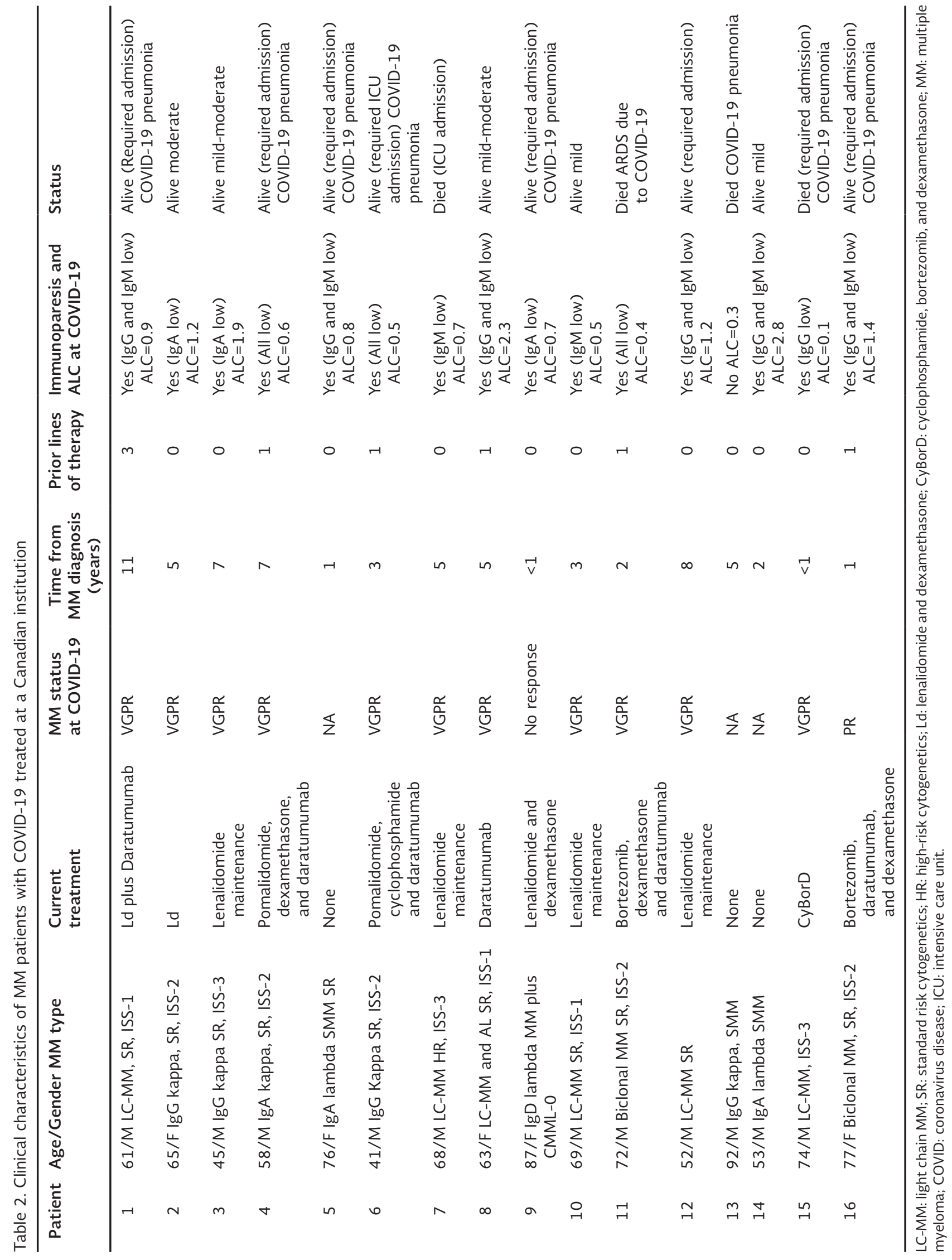


and no cause of death is reported yet for the remaining 24 patients. This represents an increase of deaths as compared to the same time period from 2019 to $2020(n=35)$ and 2018-2019 ( $n=47)$.

Furthermore, a cohort of recently diagnosed MM patients treated and followed at TBCC was analyzed. A total of 324 patients were evaluated, 257 (79.3\%) are alive and 243 (75\%) have not progressed. Median overall survival (OS) has not been reached. From those who had died, 29 died in the COVID-19 pandemic era with a median OS of 19 months. Most of these cases have progressed and likely died with active myeloma ( $57 \%$ ). About $25 \%$ of the death cases were seen in patients with high-risk MM defined by FISH cytogenetics $(\mathrm{t}(4 ; 14), \mathrm{t}(14 ; 16)$, and $17 \mathrm{p}$ deletion), $24 \%$ had elevated lactate dehydrogenase levels, $79 \%$ were ISS2-3, and two more presented with extramedullary disease at the time of diagnosis (6.8\%). In this cohort, $40 \%$ were tested for COVID-19 and 3 (2.3\% of tested cases) of them resulted positive; one patient died due to COVID-19 pneumonia.

\section{DISCUSSION}

The COVID-19 pandemic, caused by the novel SARSCoV-2 virus, has resulted in over 600,457 deaths in the United States and 26,368 in Canada (https:// covid19.who.int). In Alberta, 2,305 deaths have been registered (July 7,2021 ). The average age at death is 80 years, with 232, 269 cases reported across the province (https://www.canada.ca/en/public-health/ services/diseases/2019-novel-coronavirus-infection. html).

\section{COVID-19 and cancer}

Initially, Liang et al. reported a cancer prevalence of $1 \%$ among the 1,590 patients with COVID-19 infection ${ }^{2}$. This represents a higher rate as compared with the overall cancer incidence of $0.29 \%$ in the Chinese population ${ }^{6}$. Further, a recent study from the UK Coronavirus Cancer Monitoring Project (UKCCMP) compared a cohort of cancer patients enrolled in the study with a parallel non-COVID-19 cancer control population. The study showed $30.6 \%$ death rate in the UKCCMP cohort ${ }^{7}$. Patients with hematological malignancies (lymphoma, leukemia, and myeloma) had a more severe COVID-19 trajectory compared with patients with solid tumors (OR 1.57). As a result, many cancer organizations implemented recommendations for SARS-CoV-2 screening among patients, staff, and providers, as well as other policies aiming to minimize time in hospital, laboratory facilities, and use of telehealth resources ${ }^{8-13}$.

\section{Local experience}

Here, we present a comprehensive report of the impact of COVID-19 for the management of MM at a Western Canadian plasma cell disorders referral center. First, we present data on the rate of new diagnosis of MM. It is important to note that we observed a decrease in the number of patients diagnosed with both SMM and active MM in 2020 as compared with the previous year (21.7\% less for SMM and $22.8 \%$ for active MM). According to Alberta Health Services, the number of new cancers diagnosed between March and October was $20 \%$ lower than the same time period in 2019 and here we have confirmed this data to be true for patients with MM.

Second, we report a cohort of newly diagnosed MM patients from 2019 to 2020 aiming to evaluate differences in clinical presentation features. The current study revealed that patients with new diagnosis of MM in 2020 were clinically similar to those seen in 2019 except for more tumor burden and an increase of extramedullary disease. This latter feature should be cautiously interpreted as we do not routinely perform PET/CT scans for all newly diagnosed MM patients, and this could be a misrepresentation of the real frequency of extramedullary disease at the initial myeloma presentation.

Transplant-related mortality for MM patients was similar to previous years, and we could offer transplants to all MM patients who were eligible with no major delays. In contrast, clinical trial enrollment decreased $16.6 \%$ in 2020 as compared to the previous year (2019) as a result of delays in opening new trials or pauses on enrollment for some of the ongoing studies at our center. On the other hand, treatment dispensation patterns or progression rates were similar in 2020 and 2019 as per our pharmacy database.

Finally, we report 16 patients with MM who developed COVID-19 infection, 2 with SMM on observation and 
14 cases with active MM treated with different regimens, including daratumumab-based combinations in six patients, $\mathrm{PI}$ in three, and ImIDs in nine cases. Our report is in agreement with those that showed a higher fatality rate for patients with MM that had developed COVID-19 infection ${ }^{4,14-17}$. About $25 \%$ of our patients died as a consequence of COVID-19 infection and 10 patients developed COVID-19 pneumonia.

Estimates of excess deaths can provide information about the burden of mortality potentially related to the COVID-19 pandemic, including deaths that are directly or indirectly attributed to COVID-1918. Based on the above, we looked at $886 \mathrm{MM}$ patients diagnosed from 2011 to present to assess excess of deaths in MM. We noted an excess of deaths in the pandemic year compared to 2019 ( 57 vs. 35 deaths). Cause of death has not yet been reported in a proportion of patients who died in the time of the pandemic. However, $40 \%$ of the deaths were associated with myeloma disease progression, and $17.5 \%$ were not associated with myeloma-related events.

COVID-19 pandemic has affected the way we live and has had a tremendous impact on cancer diagnosis, prognosis, and therapeutic effects. Emerging studies show a worse trend among cancer COVID-19 patients, including patients with $\mathrm{MM}^{5,19}$.

In conclusion, the present study confirms that patients with $\mathrm{MM}$ are vulnerable and exhibit a higher fatality rate if infected with COVID-19. COVID-19 has posed one of the greatest challenges for our health-care system in recent years, and $\mathrm{MM}$ patients are not excluded from the impact of the pandemic. An excess of deaths in MM and a lower rate of myeloma diagnosis are reported in the current study. Since MM patients are more susceptible to develop severe complications from COVID-19, it is also important to make sure that there is a good support system to diagnose and manage the impact of COVID-19 on MM.

\section{REFERENCES}

1. Guan WJ, Ni ZY, Hu Y, Liang WH, Ou CQ, He JX, et al. Clinical characteristics of coronavirus disease 2019 in China. N Engl J Med. 2020;382:1708-20.

2. Liang W, Guan W, Chen R, Wang W, Li J, Xu K, et al. Cancer patients in SARS-CoV-2 infection: a nationwide analysis in China. Lancet Oncol. 2020;21:335-7.

3. Jimenez-Zepeda VH, Duggan P, Neri P, Chaudhry A, Tay J, Bahlis N. Immunoparesis and polyclonal immunoglobulin recovery after auto-SCT for patients with multiple myeloma treated at a single institution. Leuk Lymphoma. 2018;59:1920-6.

4. Chari A, Samur MK, Martinez-Lopez J, Cook G, Biran N, Yong K, et al. Clinical features associated with COVID-19 outcome in multiple myeloma: first results from the international myeloma society data set. Blood. 2020;136:3033-40.

5. Martinez-Lopez J, Mateos MV, Encinas C, Sureda A, HernandezRivas JA, de la Guia AL, et al. Multiple myeloma and SARS-CoV-2 infection: clinical characteristics and prognostic factors of inpatient mortality. Blood Cancer J. 2020;10:103.

6. Zheng RS, Sun KX, Zhang SW, Zeng HM, Zou XN, Chen R, et al. Report of cancer epidemiology in China, 2015. Zhonghua Zhong Liu Za Zhi. 2019;41:19-28.

7. Lee LY, Cazier JB, Angelis V, Arnold R, Bisht V, Campton NA, et al. COVID-19 mortality in patients with cancer on chemotherapy or other anticancer treatments: a prospective cohort study. Lancet. 2020:395:1919-26.

8. Foley R, Kaedbey R, Song K, Venner CP, White D, Doucette S, et al. Canadian perspective on managing multiple myeloma during the COVID-19 pandemic: lessons learned and future considerations. Curr Oncol. 2020;27:270-4.

9. Hus I, Salomon-Perzynski A, Tomasiewicz K, Robak T. The management of hematologic malignancies during the COVID-19 pandemic. Expert Opin Pharmacother. 2021;22:565-82

10. Jethava YS, Fonseca R, Landgren O. Management of multiple myeloma during COVID-19 pandemic. Leuk Res Rep. 2020; 14:100212.

11. Manasanch EE, Mulanovich V, Manzano JG, Gaeta MS, Becnel M, Kaufman GP, et al. SARS-CoV-2 in multiple myeloma: initial observation and management. Leuk Lymphoma. 2020;61:2763-6.

12. Sharpley FA, Larham J, Haines A, Djebbari F, Tseu B, Leary H, et al. Myeloma clinical outcomes following the first wave of $\mathrm{CO}$ VID-19: Results from the Thames Valley Cancer Alliance (UK). Br J Haematol. 2021:192:e136-9.

13. Terpos E, Engelhardt M, Cook G, Gay F, Mateos MV, NtanasisStathopoulos I, et al. Management of patients with multiple myeloma in the era of COVID-19 pandemic: a consensus paper from the European myeloma network (EMN). Leukemia. 2020; 34:2000-11

14. Dhakal B, D’Souza A, Chhabra S, Hari P. Multiple myeloma and COVID-19. Leukemia. 2020;34:1961-3.

15. Dufour I, Raedemaeker J, Andreozzi F, Verstraete G, Bailly S Delforge M, et al. COVID-19, impact on myeloma patients. Ann Hematol. 2020;99:1947-9.

16. Engelhardt $M$, Shoumariyeh $K$, Rosner A, Ihorst $G$, Biavasco $F$ Meckel K, et al. Clinical characteristics and outcome of multiple myeloma patients with concomitant COVID-19 at comprehensive cancer centers in Germany. Haematologica. 2020;105:2872-8.

17. Hultcrantz M, Richter J, Rosenbaum C, Patel D, Smith E, Korde $\mathrm{N}$, et al. COVID-19 infections and outcomes in patients with multiple myeloma in New York city: a cohort study from five academic centers. medRxiv. 2020;2020:20126516.

18. Rossen LM, Branum AM, Ahmad FB, Sutton P, Anderson RN. Excess deaths associated with COVID-19, by age and race and ethnicity United States, January 26-October 3, 2020. MMWR Morb Mortal Wkly Rep. 2020;69:1522-7.

19. COVID-19 prolonged in patients with cancer. Cancer Discov. 2021;11(5):OF7 